\title{
The Influence of Thyroid Hormone Deficiency on the Development of Cochlear Nonlinearities
}

\author{
Lei Song, ${ }^{1,2,3}$ JoAnn McGee, ${ }^{1,2}$ and Edward J. Walsh ${ }^{1,2}$ \\ ${ }^{1}$ Developmental Auditory Physiology Laboratory, Boys Town National Research Hospital, 555 North 30th Street, Omaha, \\ NE 68131, USA \\ ${ }^{2}$ Department of Biomedical Sciences, Creighton University School of Medicine, 2500 California Plaza, Omaha, NE 68178, USA \\ ${ }^{3}$ Present address: Department of Surgery, Yale University, 333 Cedar St., BML246, New Haven, CT 06510, USA
}

Received: 12 September 2007; Accepted: 11 September 2008; Online publication: 15 October 2008

\begin{abstract}
It is well known that failure to treat severe congenital hypothyroidism leads to profound auditory disability, and it has been suggested that an intracochlear defect, or defects, associated with the condition diminishes the efficacy of an active, physiologically vulnerable nonlinear transduction process commonly referred to as cochlear amplification. We address this question directly by tracking the development of threshold-frequency (tuning) curves and two-tone suppression in hypothyroid, Tshr mutant mice born to hypothyroid dams and comparing those findings with findings observed in euthyroid mice. Like sharp tuning, two-tone suppression is a product of transduction nonlinearity and is a useful indicator of the functional status of cochlear amplification. In contrast to euthyroid mice that acquire sharp tuning, normal two-tone suppression, and adultlike sensitivity by the end of the third postnatal week, as shown in earlier studies, hypothyroid mice remained grossly insensitive to sound throughout life. In addition, tuning was generally broad in hypothyroid mice, tuning curve "tips" were frequently missing, and two-tone suppression was rarely observed. However, unlike tip thresh-
\end{abstract}

Correspondence to: Edward J. Walsh - Developmental Auditory Physiology Laboratory · Boys Town National Research Hospital . 555 North 30th Street, Omaha, NE 68131, USA. Telephone: +1402-4986701; fax: +1-402-4986351; email: walsh@boystown.org olds, tuning curve "tail" thresholds, a feature that reflects the functional status of passive elements of transduction, acquired normal values over a roughly 2-month postnatal time period. These observations collectively suggest that active transduction micromechanics, at least in the frequency region studied here, are profoundly affected by thyroid hormone and support speculation that abnormal outer hair cell function may be the cause of the primary, enduring peripheral auditory defect associated with profound, congenital hypothyroidism in the Tshr mutant mouse.

Keywords: hypothyroidism, cochlear amplifier, frequency selectivity, two-tone suppression, transduction, cochlea, auditory nerve

\section{INTRODUCTION}

A paradox of sorts has arisen in the literature regarding the extent of peripheral auditory abnormality associated with untreated, profound congenital hypothyroidism. The anatomical record accumulated from various laboratories suggests that cochlear abnormalities are widespread among congenitally hypothyroid individuals and involve structures in both the middle and inner ears (see Walsh and McGee 2001 for a review). Physiological findings, in contrast, suggest that the primary enduring peripheral auditory defect may be more limited (Walsh and McGee 2001; Song et al. 2006a). 
In that context, the outcome of a recent investigation (Song et al. 2006a) revealed a previously unreported developmental stage among hypothyroid, $T s h r^{h y t / h y t}$ mutant mice ${ }^{1}$ born to hypothyroid dams that extends into the second and possibly third postnatal month. Although untreated mutant animals remained seriously impaired throughout the age range studied (i.e., beyond 90 postnatal days), sensitivity to tone bursts improved as much as $30 \mathrm{~dB}$ during a roughly 2-month postnatal period, and circumstantial evidence suggested that the gain of the cochlear amplifier may have been reduced in affected animals.

Because passive and active elements of cochlear transduction reach maturity sequentially according to the most widely accepted developmental models (Henley et al. 1990; Lenoir and Puel 1987; Norton et al. 1991; Fitzakerley et al. 1994a, b, c; Tubach et al. 1996; Mills and Rubel 1996), we propose to test the hypothesis that unlike active aspects of transduction, biomechanical features associated with passive transduction develop normally during this protracted postnatal period in profoundly hypothyroid mice, at least in the frequency region studied here. We address this question from a functional viewpoint by tracking developmental changes in tuning curves (threshold vs. frequency curves) derived from wave I of the auditory brainstem response (ABR) using a forwardmasking procedure in profoundly hypothyroid mice and compare those findings with findings obtained from euthyroid mice.

In that regard, Song et al. (2008) showed that tuning curves derived from wave I of the ABR in the adult mouse are very similar to those derived from the responses of auditory nerve fibers (Taberner and Liberman 2005). It is possible, therefore, to evaluate the development of passive and active transduction mechanics by considering the maturation of lowfrequency "tail" thresholds of tuning curves and the most sensitive "tip" thresholds separately. Tuning curve tails are known to reflect the linear, insensitive, and passive aspect of cochlear transduction, while tuning curve tips reflect the coordinated activity of both passive and active processes. Consequently, the magnitude or gain of the cochlear amplifier can be

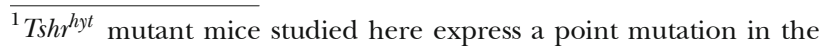
gene that encodes the thyrotropin receptor $(T s h r)$ and the phenotype of individuals that are homozygous for the hyt trait is profound thyroid hypoplasia and hypothyroidism. The thyrotropin receptor belongs to the G-protein class of membrane-spanning proteins, and the amino acid substitution resulting from this mutation occurs in a highly conserved region of the receptor, in which proline is replaced by leucine at position 556 in transmembrane domain IV, transforming the protein to a form that is unable to bind thyrotropin (TSH) and producing primary congenital hypothyroidism (Gu et al. 1995; Stein et al. 1994) and associated auditory pathology as a consequence (O'Malley et al. 1995; Li et al 1999; Sprenkle et al. 2001a, b, c; Walsh and McGee 2001; Song et al. 2006a).
}

estimated by considering tip-to-tail differences, and the relative maturation of tail and tip thresholds can be used to assess the development of linear and nonlinear aspects of transduction simultaneously. This approach will support objective efforts to identify which elements of transduction (passive or active or both) are affected by profound congenital hypothyroidism and advance understanding of auditory system development in mice generally.

\section{MATERIALS AND METHODS}

\section{Animals}

All experimental subjects were bred in-house from animals that were descendents of the $T s h r^{h y t}$ colony (CBy.RF-Tshr $r^{h y t} / \mathrm{J}$ ) at The Jackson Laboratory (Bar Harbor, ME, USA). Breeders consisted of homozygous mutant $\left(T s h r^{\text {hyt } / h y t}\right)$ females paired with heterozygous $\left(T s h r^{+/ h y t}\right)$ males. To enhance fertility, hypothyroid dams were fed commercial rodent chow supplemented with desiccated porcine thyroid powder $(25 \mathrm{mg} / \mathrm{kg}$ feed) for a period of approximately 1 week prior to mating. Homozygous mutant progeny are thus deprived of endogenous thyroid hormone, as well as maternally derived thyroid hormone.

Euthyroid control animals consisted of heterozygous $\left(T s h r^{+/ h y t}\right)$ mutant and wild-type mice derived from progenitor pairings. Heterozygous mutant mice and wild-type mice were indistinguishable from one another (unpublished observations). Euthyroid and hypothyroid subjects of either gender were randomly selected for testing. Control animals were studied between postnatal day 12 (P12) and P90, and hypothyroid animals were studied between P24 and P90 because of developmentally delayed response to acoustic stimulation (Song et al. 2006a, b). The day of birth was designated as P0. Individual animals were studied longitudinally when possible. Development of cochlear nonlinearities in euthyroid animals serving as controls is described in greater detail in an earlier paper (Song et al. 2008).

The care and use of all animals involved in this study were approved by the Institutional Animal Care and Use Committee of the Boys Town National Research Hospital.

\section{Phenotyping and genotyping}

Homozygous and heterozygous animals were distinguished on the basis of both phenotype and genotype. Body weight and size are generally reliable indicators of the genetic status of young adult animals (Sprenkle et al. 2001b), and distinct differences between hypothyroid animals and their euthyroid littermates were apparent by P12, with the mean body weight of 
hypothyroid animals averaging $4.8 \pm 0.14$ vs. $8.6 \pm 1.0 \mathrm{~g}$ for euthyroid controls. Body weight differences increased as development progressed, such that by P28, mean body weights for hyt/hyt vs. +/hyt were $8.8 \pm 0.8$ and $16.8 \pm 2.0 \mathrm{~g}$, respectively. Acoustic sensitivity was also a useful indicator of phenotype, with hypothyroid individuals exhibiting clearly elevated thresholds relative to euthyroid individuals, regardless of age.

Mice derived from the $T s h r^{h y t}$ colony were also genotyped. A PCR primer pair was designed from the thyrotropin receptor sequence (Genbank accession \#U02601) using OLIGO 6.0 (Molecular Biology Insights, Cascade, CO, USA) that flanked the missense mutation. Genotypes were determined by sequencing the mouse PCR amplimers. Tail biopsies were incubated in a sodium dodecyl sulfate and proteinase $\mathrm{K}$ lysis solution overnight at $55^{\circ} \mathrm{C}$. This mixture was phenol-chloroform-extracted, and mouse genomic DNA was ethanol-precipitated. The primer pair (U02601:2475L19 5'-TTGGCAATCTTGGTGTCTT-3' and U02601:2475U19 5'-AGGCACGGGTACAC CATCA-3') was used to PCR amplify a segment of the mouse thyrotropin receptor gene. Amplification conditions were 36 cycles at $95^{\circ} \mathrm{C}$ for $30 \mathrm{~s}, 65^{\circ} \mathrm{C}$ for $30 \mathrm{~s}$, and $72^{\circ} \mathrm{C}$ for $1 \mathrm{~min}$, using AmpiTaq Gold (PE Express). PCR-amplified DNA was purified for sequencing by selective filtration using the Microcon YM-100 (Amicon/Millipore). DNA sequencing was accomplished using the ABI Prism 377 DNA Sequencer and the ABI Prism BigDye Terminator Sequencing Reaction Kit. Sample files were generated from gel files using ABI Sequencing Analysis 3.0.

\section{Animal preparation}

Animals were anesthetized with chloral hydrate (480 mg/kg, IP), and supplemental doses (120 mg/kg) were administered as needed, with younger animals receiving lower dosages $(240-360 \mathrm{mg} / \mathrm{kg}$ initial dose and $90-120 \mathrm{mg} / \mathrm{kg}$ supplemental doses). Body temperature was thermostatically regulated and maintained at approximately $38.5^{\circ} \mathrm{C}$ throughout recording sessions. Subdermal needle electrodes (Grass Instruments) were positioned at the vertex (active, noninverting), infraauricular mastoid region (reference, inverting), and in the neck region (ground). All recordings were conducted in a double-walled sound-attenuating chamber (Industrial Acoustics).

\section{Acoustic system and calibration}

Two independent channels of the sound system were used to deliver the stimuli. Computer-generated digital waveforms were transformed by 16-bit digital to analog converters. Stimuli were amplified by a lowdistortion amplifier (Crown D75) and attenuated using custom-built attenuators with a range of $127 \mathrm{~dB}$. Free-field sound stimuli were delivered through two high-impedance piezoelectric tweeters (Radio Shack) positioned along the animal's midline and placed $10 \mathrm{~cm}$ from the vertex. Tone bursts were symmetrical and ramped with 1-ms cosine-shaped rise and fall times. Probe stimuli had a 1-ms plateau and were $3 \mathrm{~ms}$ in duration, whereas masker and suppressor stimuli were $50 \mathrm{~ms}$ in duration. Stimulus levels were calibrated using a 0.5-in. Brüel and Kjær microphone (Model 4134) positioned at the approximate location of the subject's head during recordings sessions and are reported in decibels sound pressure level (dB SPL: referenced to $20 \mu \mathrm{Pa}$ ).

\section{Recording procedure}

ABRs were recorded differentially using standard procedures (Song et al. 2006b). Voltages recorded from the scalp were amplified 100,000× using a Grass P511 preamplifier, band-pass-filtered between 0.03 and $10 \mathrm{kHz}$, and digitized at a $20-\mathrm{kHz}$ sampling rate over a 15-ms epoch using a Cambridge Electronics (CED 1401 plus) A/D converter. Trials containing peak-to-peak voltages exceeding $70 \mu \mathrm{V}$ were automatically rejected, and the trial was repeated. A total of 200 trials were averaged for each stimulus condition using customized software to acquire evoked potentials.

In a subset of animals studied here, ABR thresholds from 2 to $32 \mathrm{kHz}$ were obtained in half-octave steps, and level series were obtained at each of these frequencies in 10-dB steps, except near threshold where level was changed in 5-dB steps. The results of those analyses are included in a previous report (Song et al. 2006a). For the remaining animals, threshold and a level series were obtained in response to the probe frequency, prior to the acquisition of tuning curves and suppression areas (Fig. 1A,C).

\section{Acquisition of tuning curves}

A forward-masking procedure was used to derive tuning curves from scalp-recorded evoked potentials based on the technique developed by Dallos and Cheatham (1976) and described in greater detail in Song et al. (2008). Probe stimuli at either 8 or $11.3 \mathrm{kHz}$ were presented approximately $5-15 \mathrm{~dB}$ above threshold to produce a control response of approximately $1.3 \mu \mathrm{V}$. A masker tone burst was presented prior to the probe, with a 10-ms interval $(\Delta t)$ separating masker offset and probe onset. Masker level was varied in 5-dB steps, or less, from threshold to a level that reduced the potential (wave I amplitude) elicited by the probe by more than $50 \%$ (Fig. 1B, D). The amplitude of wave I of the ABR was quantified using a triangulation procedure (Walsh et al. 1986) and plotted as a function of masker level. 

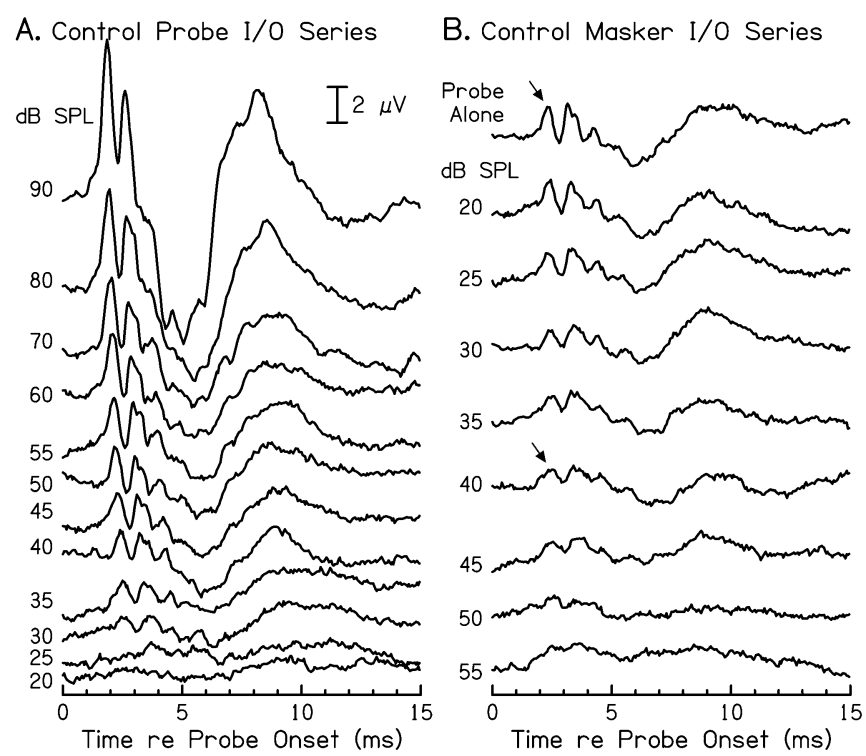

FIG. 1. Frequency-threshold (tuning) curves were derived from the $A B R$ using a forward-masking paradigm. ABRs were initially acquired at the probe frequency from below threshold to suprathreshold levels as shown for a control (A) and hypothyroid mouse (C). From these data, the level of the probe stimulus that elicited a criterion wave I amplitude was determined; waveforms in response to the "probe alone" condition are shown at the top of $\mathbf{B}$ and $\mathbf{D}$, respectively. The effects of a masker signal presented at different levels on the response to the probe were then assessed for the control (B) and hypothyroid

The level of the masker that produced a $50 \%$ reduction of the probe-elicited response was computed by linear interpolation and defined as masked threshold. Masked thresholds were then used to construct a tuning curve by plotting those levels (i.e., thresholds) as a function of masker frequency (Fig. 1E,F). Approximately three to five masker tones separated by $1 / 8$ th octave intervals with center frequencies above the probe tone and approximately four maskers separated by $1 / 8$ th octave intervals with center frequencies below the probe frequency, followed by an additional four to five maskers at more distant frequencies separated by $1 / 4$ th octave intervals were used in acquiring each tuning curve. Probe-alone conditions were regularly interleaved with masker conditions to assure that the amplitude of the control response did not vary significantly during the recording session.

Tip thresholds were determined by recording the value observed at the probe frequency, and tail thresholds were determined at 1.0 octave below the probe frequency in the case of the $8-\mathrm{kHz}$ probe and at 1.5 octaves below the probe frequency for the 11.3$\mathrm{kHz}$ condition. Sharpness of tuning was computed by dividing the probe frequency by the bandwidth measured $10 \mathrm{~dB}$ above threshold at the probe frequency $\left(Q_{10}\right)$. Finally, tip-to-tail ratio was determined by computing the difference in decibels between tip and tail thresholds.
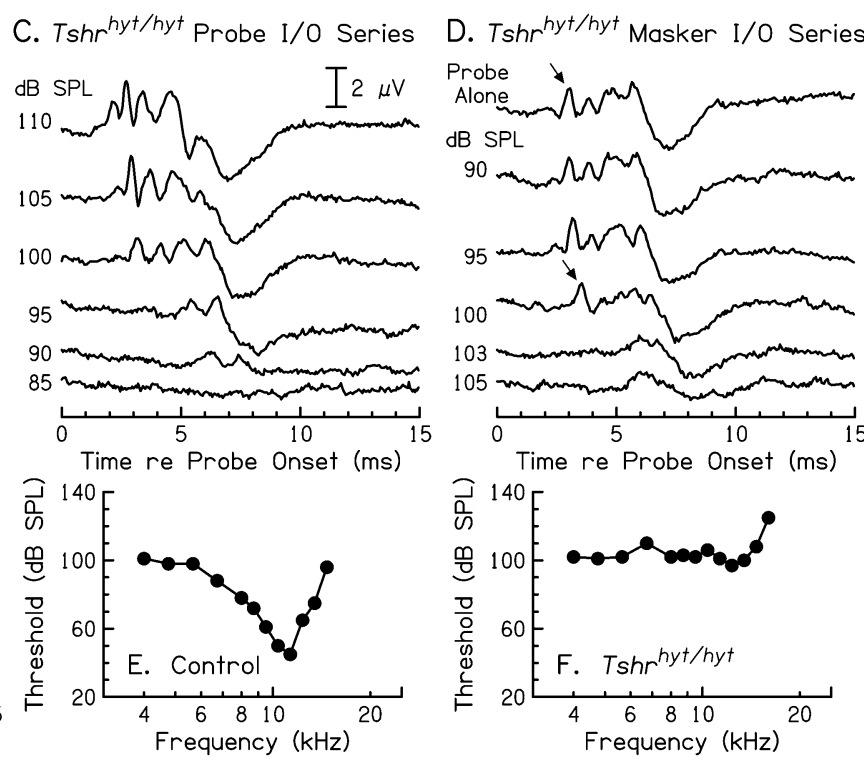

mouse (D). The level of the masker that reduced the amplitude of wave I elicited in response to the probe by $50 \%$ was plotted as a function of masker frequency as shown in $\mathbf{E}$ and $\mathbf{F}$, respectively. Note that the amplitude scale shown in $\mathbf{A}$ applies to $\mathbf{A}$ and $\mathbf{B}$, and the scale in $\mathbf{C}$ applies to $\mathbf{C}$ and $\mathbf{D}$. Probe and masker frequencies were $11.3 \mathrm{kHz}$. Data shown were obtained from a control at P25 and a hypothyroid mouse at P62. Arrows in B and D identify wave I for the control condition and for the masked waveform nearest to the level producing $50 \%$ amplitude reduction.

\section{Measurement of two-tone suppression}

"Two-tone" suppression was estimated by measuring the degree to which a probe response was released from the influence of a masker tone based on the technique used by Harris (1979) and described in greater detail in Song et al. (2008). The duration and relative timing of the masker and probe tones were identical to those used to acquire tuning curves, and a third tone, the suppressor, was gated on and off at the same time as the masker tone. The level of the probe tone was maintained at the same level used to acquire the tuning curve, and the masker was equal in frequency to that of the probe and adjusted to the lowest level producing $100 \%$ masking. Suppressor level was varied, and the amount of suppression produced by suppressor tones placed at frequencies above and below the probe frequency was computed as the fraction of the probeelicited response that was released from masking.

\section{Data analysis}

Tuning curve tip and tail thresholds, tuning sharpness $\left(Q_{10}\right)$, and tip-to-tail ratios were plotted as a function of postnatal age for each probe frequency. The changes associated with each parameter during development were described by least squares linear fits in the form of $y=a+b x$. For euthyroid animals, lines were fit to 
values extending throughout an arbitrarily determined linear phase between P12 and P15 and P12 and P14.5 for tip and tail thresholds, respectively; in the case of tail thresholds, the average value on P15 was slightly higher than the average on P14.5 and was consequently excluded from the regression. Likewise, in the case of tip-to-tail ratios, regressed values were restricted to measurements made between P13 and P15, because values remained constant until P13. Similar restrictions were applied in the case of tuning curve sharpness (measurements were made between P13.5 and P15) for the same reason (see Song et al. 2008). In the case of hypothyroid animals, because of developmental delays, data were fitted to all values $\leq$ P64. In addition, data from euthyroid animals were described by an exponential fit in the form of $y=a+b e^{-c X}$, where $a$ denotes asymptote, the sum of $a$ and $b$ is the $y$ intercept, and the reciprocal of $c$ is the time constant.

The significance of each regression was tested using an ANOVA approach, and comparisons of the adult values and developmental rates were made for each probe frequency. Results were considered statistically significant when $P<0.001$, unless otherwise specified.

\section{RESULTS}

A series of ABR waveforms elicited by $11.3-\mathrm{kHz}$ tone bursts at stimulus levels selected throughout the dynamic response range are shown in Figure 1 for both control (A) and hypothyroid (C) mice. Waveform morphologies were similar in both cases, exhibiting four or five positive-going peaks that occurred within the first $6 \mathrm{~ms}$ or so following the onset of stimulation and a broad peak that was commonly observed between $\sim 6$ and $10 \mathrm{~ms}$, most notably in response to higher level tone bursts. Likewise, ABR waveforms elicited from hypothyroid mice (Fig. 1D) in response to probe stimuli that followed a masking tone (i.e., the so-called forward-masking protocol) were comparable to waveforms elicited under forwardmasking conditions from control animals (Fig. 1B), regardless of level. Although masker tones were ineffective at low levels, at higher levels, peak probe response amplitudes decreased directly and systematically as masker level increased. As expected given the steep slope of wave I amplitude-level curves observed in Tshr mutant mice (Song et al. 2006a), the dynamic range of masking was also constrained under conditions of hypothyroidism (Fig. 1D). Under these conditions, response amplitude decreased from nearly normal to an undetectable level abruptly, often within a few decibels, unlike that observed in control animals (Fig. 1B).

Tuning curves were derived from ABRs using the forward-masking protocol described in Figure 1. As with altricial mammals generally (see Walsh and
Romand 1992 and Rubsamen and Lippe 1998 for reviews), tuning curves recorded from normal, neonatal mice were primitive, exhibiting band-pass-like characteristics with little or no evidence of highfrequency tips (see Song et al. 2008 for the details of normal development). However, mature tuning was acquired rapidly in normal mice, with tip thresholds tracking along an exponential time course and achieving maturity by roughly P19. Tail thresholds achieved maturity by $\sim \mathrm{P} 15$, approximately 4 days earlier than did tip thresholds.

The development of tuning in the case of hypothyroid animals was dramatically different than in normal animals. While tuning curves were fully mature by P24 among euthyroid animals, they remained grossly immature among Tshr mutant mice, as shown in the form of individual examples in A and C of Figure 2. Tuning curves were sufficiently similar at each age studied to permit pooling, as indicated by the relatively small degree of variance from case to case, and the average tuning curves for both stimulus conditions studied are shown in B and D of Figure 2. While thresholds improved slightly between P24 and P60 within the hypothyroid population, tuning curves remained fairly flat and appeared immature, resembling tuning curves obtained from control animals between P13 and P14. Thresholds in hypothyroid mice were grossly elevated with just the slightest evidence of a tip at older ages, and tip-to-tail ratios were generally below $10 \mathrm{~dB}$, and in some cases, tail thresholds were below tip thresholds.

To more fully appreciate the developmental difference between hypothyroid and euthyroid animals, tip thresholds, tail thresholds, tip-to-tail ratios, and tuning sharpness were tracked throughout development (i.e., to the 90th postnatal day; Fig. 3). While maturation can be reasonably characterized as an exponential process achieving $95 \%$ of asymptotic values by $\sim \mathrm{P} 15-$ P21 for each of these parameters in normal animals, tuning curves from Tshr mutant mice changed over a protracted time period and followed a linear trajectory. Parametric changes in tuning curve features appeared to stabilize by $\sim \mathrm{P} 64$, albeit at values that would be regarded as unambiguously abnormal, except in the case of tail thresholds. While it is clear that tip thresholds (A), tip-to-tail ratios (C), and sharpness of tuning (D) remain significantly elevated, reduced, and broad, respectively, tail thresholds (B) acquired nearly normal values by $\mathrm{P} 64$.

The dramatic differences in tuning curve properties that distinguish normal and mutant animals are easily visualized in Figure 4. Tip and tail thresholds, amplifier gain (tip-to-tail ratio), and ABR thresholds for both $8 \mathrm{kHz}$ (left column) and $11.3 \mathrm{kHz}$ (middle column), as well as $Q_{10}$ values (right column), are shown for adult control and mutant animals in the 

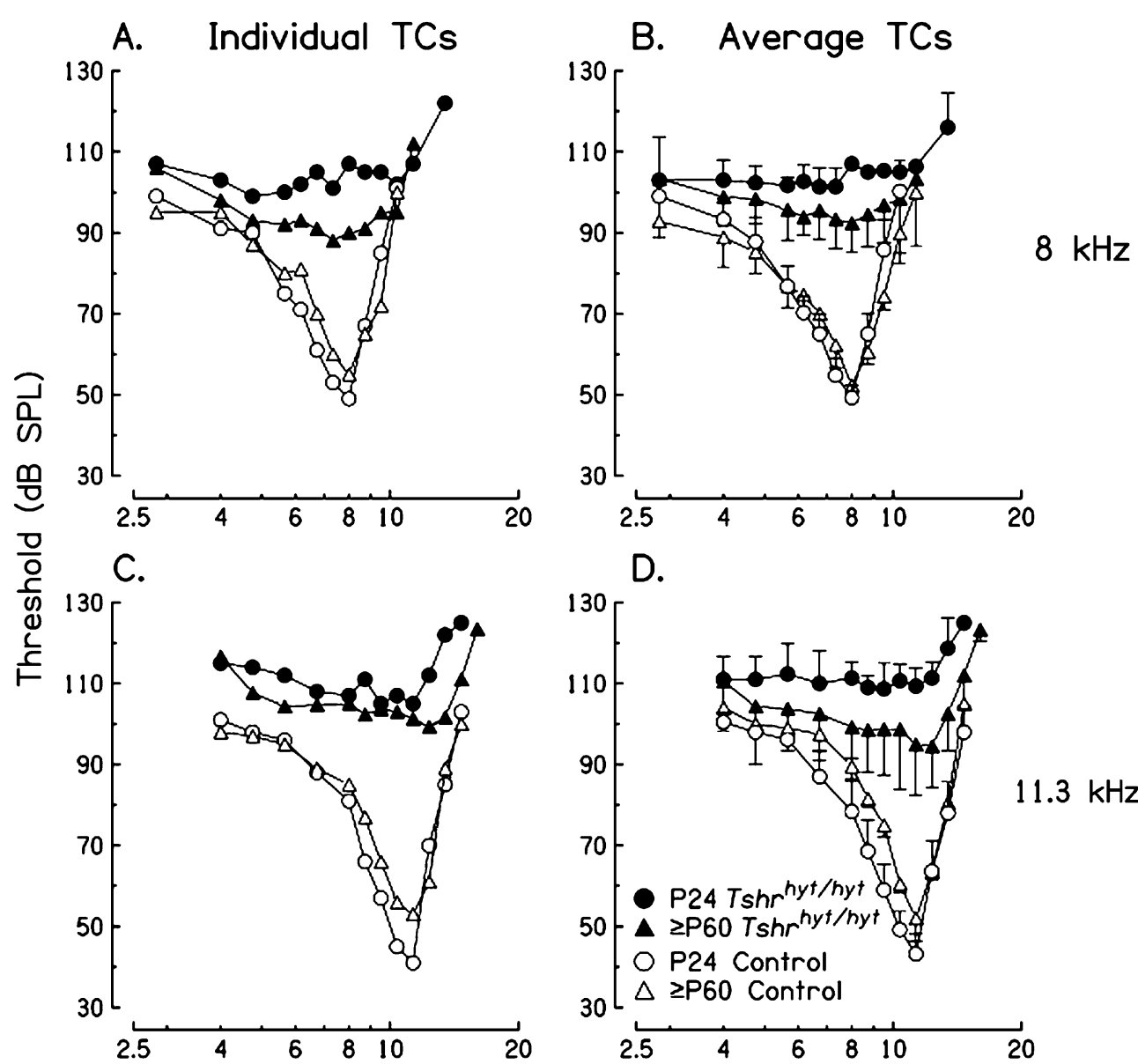

D.

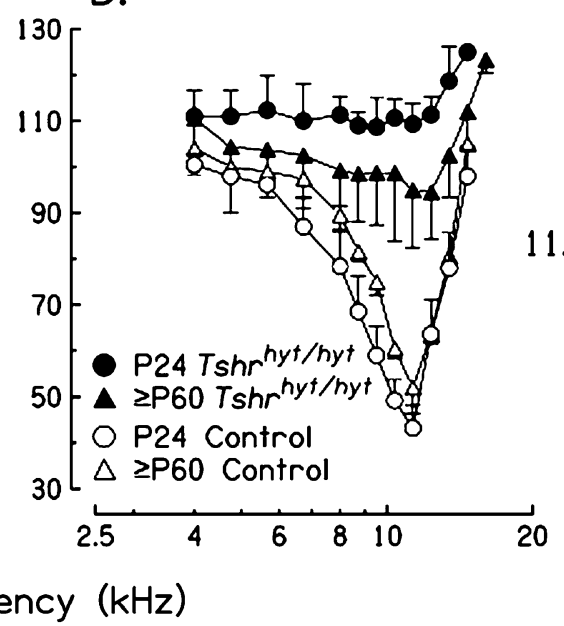

$11.3 \mathrm{kHz}$

Frequency $(\mathrm{kHz})$

FIG. 2. Tuning curves with characteristic frequencies at $8 \mathrm{kHz}$ (upper panels) and $11.3 \mathrm{kHz}$ (lower panels) are shown for control (unfilled symbols) and hypothyroid animals (filled symbols) at the indicated ages. Tuning curves from individuals are plotted in $\mathbf{A}$ and $\mathbf{C}$, and averages are shown in B and D. Error bars represent \pm 1 standard deviation and are plotted in a single direction for purposes of clarity.

top row of Figure 4. Differences between control and hypothyroid animals were statistically significant $(P<$ 0.001 ) for tip thresholds, amplifier gain, and tuning sharpness for each probe frequency studied. However, significant differences were not observed in adult tail thresholds at $11.3 \mathrm{kHz}$, and thresholds at $8 \mathrm{kHz}$ were slightly higher than normal (i.e., $\sim 2 \mathrm{~dB}$ ) in hypothyroid animals $(P<0.05)$. Maturity, defined as the age at which $95 \%$ of adult, or asymptotic values were achieved, was clearly extended in hypothyroid animals, being delayed by approximately 1.5 months on average for each tuning curve feature studied (Fig. 4D-F). Finally, developmental rate differences between the two groups were objectively determined by comparing the slopes of best-fitting lines to the linear segment of response vs. age curves. As shown before (Song et al. 2008), response growth was rapid between P12 and P15 in normal, control mice, whereas developmental rates were significantly slower in Tshr mutant mice than in controls for all tuning curve properties (Fig. 4G-I).
Additional evidence that transduction dynamics are primarily limited to passive events in Tshr mutant mice was acquired as a result of efforts to assess the development of two-tone suppression. In normal mice, suppression first appears in concert with relatively sharp tuning curve tips, above and below the characteristic frequency $(\mathrm{CF})$ at roughly the same developmental stage, and grew in strength with age and in proportion to tuning curve tip sensitivity and sharpness (Song et al. 2008). Unlike euthyroid mice, two-tone suppression was not observed in hypothyroid animals (Fig. 5), even among animals as old as P90.

\section{DISCUSSION}

While many inner and middle ear anomalies have been reported in hypothyroid animals, the most commonly and consistently reported defects are limited to those associated with the tectorial membrane (TM) and cochlear innervation (Deol 1973, 


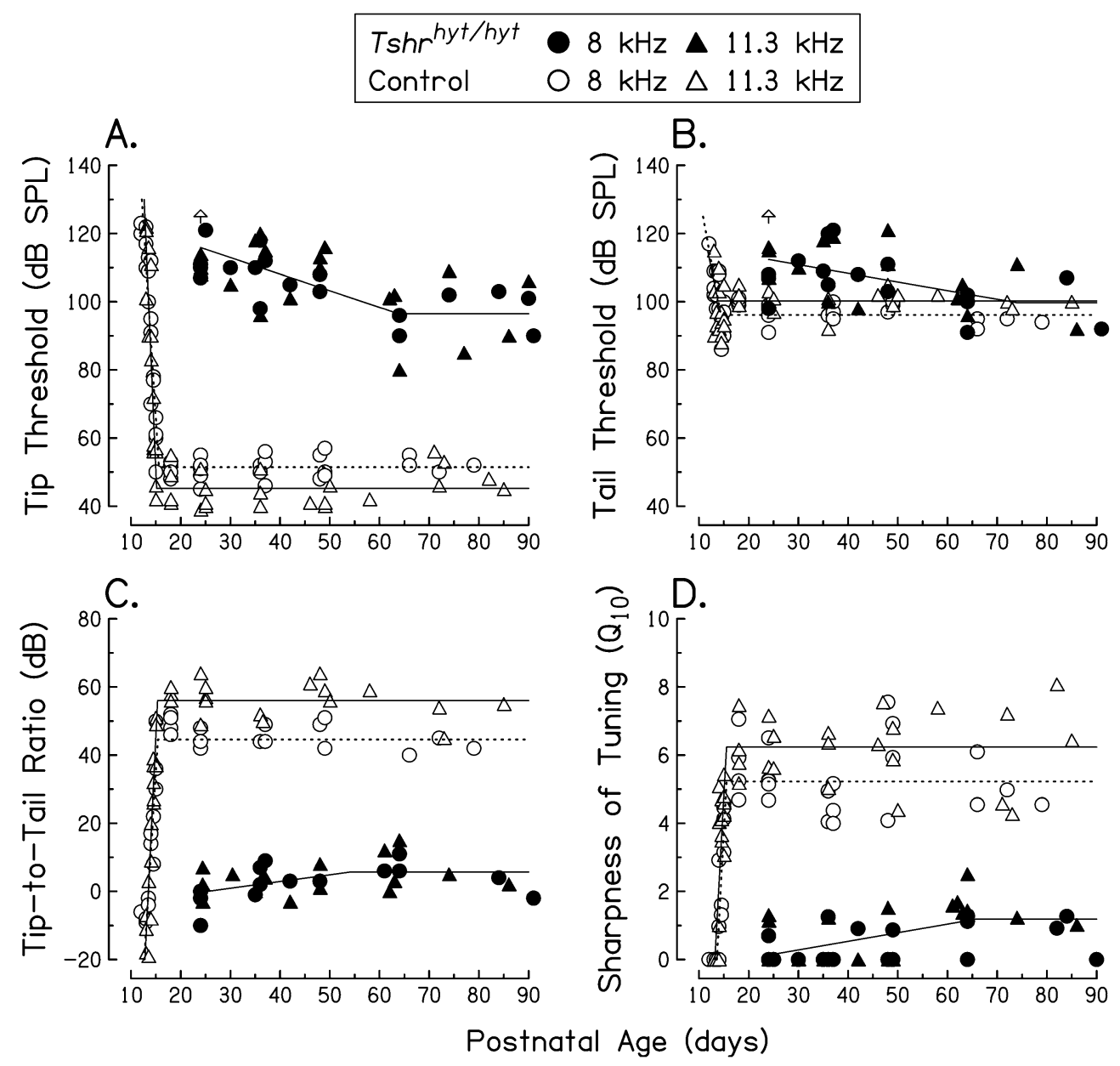

FIG. 3. The development of tuning curve tip thresholds (A), tail thresholds (B), tip-to-tail ratios $(\mathbf{C})$, and tuning sharpness measured using $Q_{10}$ (D) are shown for both control (open symbols) and hypothyroid (filled symbols) animals. Each point represents the value acquired from one animal. Probe frequencies (i.e., characteristic frequencies) were 8 and $11.3 \mathrm{kHz}$ as indicated in the symbol key.

1976; Anniko and Rosenkvist 1982; Uziel et al. 1981, 1983a, b, 1985; Remezal and Gil-Loyzaga 1993; Rusch et al. 2001; Knipper et al. 2001; Griffith et al. 2002; Christ et al. 2004; Johnson et al. 2007). Of these, the $\mathrm{TM}$ has received the greatest amount of attention as a prospective source of pathology, in part, no doubt, because of its imposing presence and distorted character, as shown in histological section under some circumstances. Nonetheless, other purportedly anomalous structures include the tunnel of Corti (Uziel et al. 1981, 1983a; Gabrion et al. 1984; Li et al. 1999), the stria vascularis (Meyerhoff 1979), the middle ear (Meyerhoff 1979; Christ et al. 2004), and sensory cells (Meyerhoff 1979; Uziel et al. 1983a; O’Malley et al. 1995; Li et al. 1999). These findings are especially interesting in the context of this study, because many of the abnormalities thus far reported involve cochlear structures that affect passive aspects of cochlear micromechanics, as well as active mecha-
Developmental data obtained for control and hypothyroid mice were fitted using least squares linear regressions. Horizontal lines represent mean adult values. Significant differences between 8 and $11.3 \mathrm{kHz}$ were not observed for responses from hypothyroid animals, and therefore, a single regression is plotted in each panel. Control values are from Song et al. (2008).

nisms. This is significant because we propose a model in which abnormalities associated with active rather than passive aspects of transduction represent the primary defect of end-stage congenital hypothyroidism.

Two key observations made in this study address what appears to be a structure/function paradox based on findings reported here and a literature suggesting that the morphological correlates of hypothyroidism involve many, if not most, peripheral auditory structures. First, approximately 1 week after normal mice achieve adultlike sensitivity and tuning, hypothyroid mice appear notably immature. Not only are tuning curve tip thresholds markedly elevated but tail thresholds are high relative to those observed in normal mice as well. Because tail thresholds reflect the functional status of passive mechanoelectrical transduction and tip thresholds the status of both passive and active mechanics, this finding suggests that the otopathology of hypothyroidism at this stage 

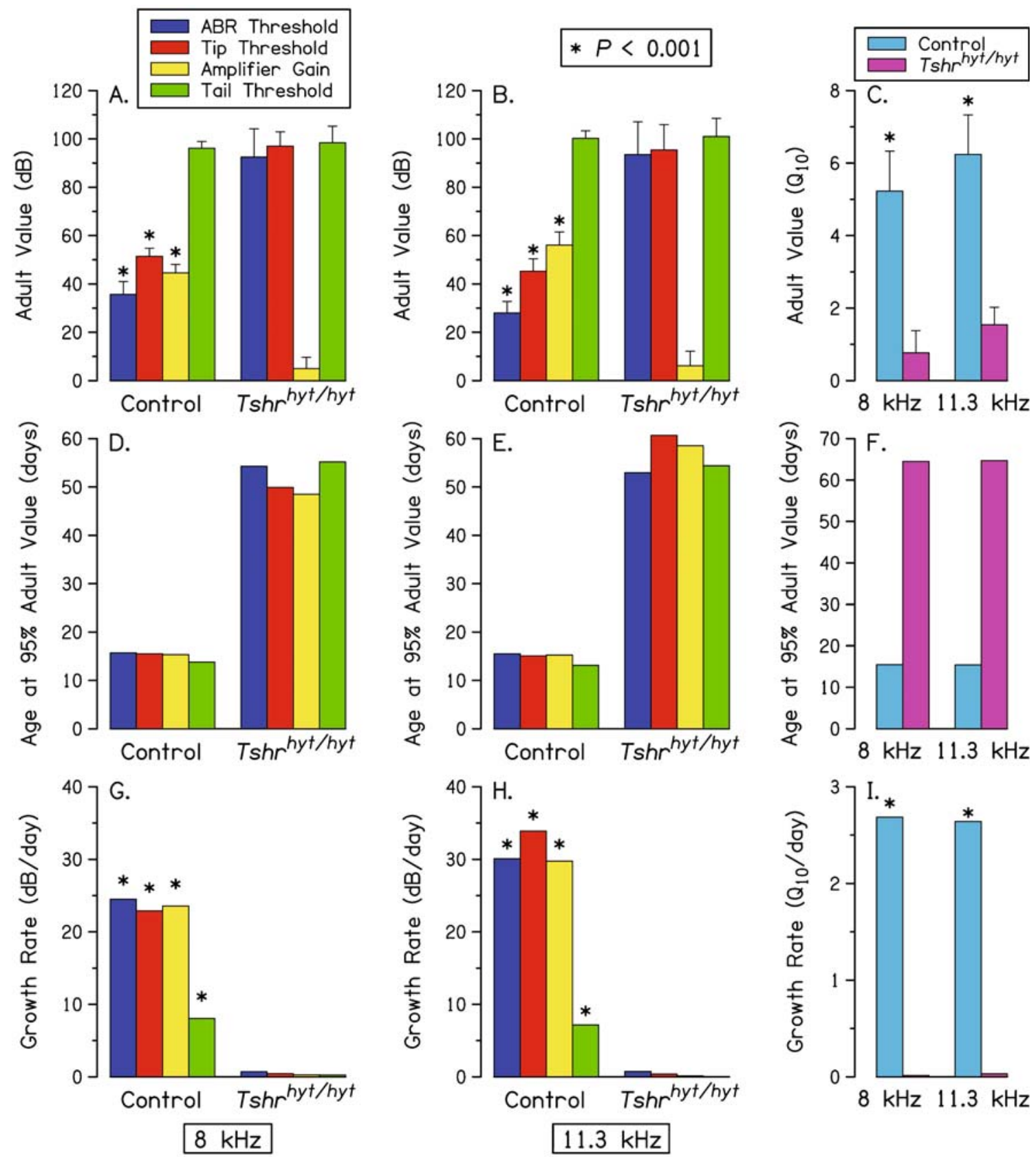

FIG. 4. Comparisons of adult values (A-C), the age at which $95 \%$ of asymptote, the adult value, was attained (D-F), and growth rates (G-I) of tuning curve tip and tail thresholds, and amplifier gain (tip-to-tail ratio), as well as ABR thresholds, are shown for control and hypothyroid (Tshr hyt/ hyt) animals at $8 \mathrm{kHz}$ (left column) and $11.3 \mathrm{kHz}$ (middle column) and for $Q_{10}$ values (right column). The key in $\mathbf{A}$ applies to all panels in the left and middle columns, whereas that shown in $\mathbf{C}$ applies to those in the right column. The asterisks indicate a statistically significant difference between values obtained for control and hypothyroid animals $(P<0.001)$. Growth rates were estimated using linear regression fits to the developmental data derived from tuning curves at each characteristic frequency ( 8 and $11.3 \mathrm{kHz}$ ). Note that in $\mathbf{G}$ and $\mathbf{H}$, the growth rates for ABR, tip, and tail thresholds represent rate of threshold improvement in decibels per day. Values for ABR threshold development are from Song et al. (2006a).

of development is compound, involving both passive and active transduction dynamics. These findings support the traditional model of the disease in which the otopathology of congenital hypothyroidism is characterized by auditory insensitivity resulting from gross organ of Corti and middle ear abnormalities. However, over the course of the following 2 months, although tip thresholds remain abnormally high and frequency selectivity remains abnormally broad, tail thresholds of hypothyroid animals gradually acquire normal or nearly normal characteristics, suggesting that the elements of passive transduction eventually achieve, or nearly achieve, maturity, while active mechanics remain decidedly abnormal in $T s h r$ mutant mice. 
The rationale underlying this conclusion is that abnormalities associated with passive aspects of transduction alter cochlear tuning in a frequency-independent manner generally, elevating both tip and tail thresholds alike (Kiang et al. 1986). It is the case, however, that hypersensitive tails and hyposensitive tips are observed when outer hair cells (OHCs) are significantly damaged and IHC stereocilia are slightly disrupted following acoustic trauma or exposure to aminoglycoside antibiotics (Liberman and Dodds 1984). If hair cell damage was observed in Tshr mutant mice, one might expect to observe hypersensitive tail thresholds. However, there is no evidence of outer or inner hair cell abnormality at the light level of microscopy in Tshr mutant mice (Walsh et al. 2007), and therefore, one would not expect to observe hypersensitive tail thresholds like those observed by Liberman and Dodds (1984). Thus, the observation that tail thresholds ultimately acquire normal or nearly normal values, while tip thresholds and frequency selectivity remain abnormal, provides fairly strong circumstantial support for the idea that an active transduction deficit is the primary source of hearing loss in untreated Tshr mutant animals.

Given the relatively wide range of peripheral auditory anomalies ostensibly associated with the disease, many of which affect passive aspects of signal processing alone (e.g., the middle ear), or both passive and active aspects of transduction micromechanics (e.g., the TM and the stria vascularis and associated endocochlear potential), findings reported here were unexpected. They can be explained, however, on the basis of two frequently overlooked, but key observations.

First, the cochlea in hypothyroid animals undergoes limited but certain development in the early postnatal period. While this is a novel and significant finding in and of itself, it is especially important in the context of this study. Because cochlear development in Tshr mutant mice plays out over a relatively protracted, two postnatal month period, and because many, if not most, hypothyroidism studies involving lab animals have been conducted at an earlier stage (e.g., $\sim 1$ postnatal month), in some cases, reported abnormalities are not abnormalities at all, but immaturities associated with delayed development. For example, although the fast-activating potassium conductance $\left(I_{\mathrm{K}, \mathrm{f}}\right)$ in inner hair cells of TR $\beta$-null mice, as well as calcium channel currents and resting membrane potentials in hypothyroid rats are developmentally delayed, adultlike values are eventually achieved (Rusch et al. 1998, 2001; Brandt et al. 2007). In OHCs of TRß-null and $T s h r^{h y t}$ mutant mice, expression patterns of the motor protein, prestin (Slc26a5), although delayed, ultimately mature (Winter et al. 2006, 2007), as do nonlinear capacitance and electromotility, respectively (Rusch et al. 1998; Walsh et al.
2003). This, of course, is not always the case. While the expression of prestin is upregulated (Weber et al. 2002) via TR $\beta$, the expression of the potassium channels, KCNQ4, BK, and SK2, for example, are actually repressed via unbound $\mathrm{TR} \alpha 1$ in the absence of TH (Winter et al. 2006, 2007) and may be the source of permanent deficits in congenital, profound hypothyroidism. These findings emphasize the importance of tracking the development of the hypothyroid cochlea to its end point.

Second, numerous lines of evidence indicate that at least some commonly identified abnormalities are transiently and/or variably expressed in animals with thyroid system pathology. The ostensibly anomalous structure of the middle ear is an ideal example of this inconsistent and variable nature of findings reported from laboratory to laboratory in hypothyroid animals. For example, while the athyroid strain of Pax8 null mice are severely hearing-impaired as a group, only a subset of animals studied by Christ et al. (2004) displayed middle ear abnormalities. Likewise, in a study of $T s h r^{h y t}$ mutant mice, O’Malley et al. (1995) reported that middle ear structures were normal. These findings suggest that factors other than abnormal middle ear function operate to affect phenotype in at least some cases of hypothyroidism.

In the same vein, although hair bundle abnormalities in the form of clumped or agglutinated stereocilia have been reported in Tshr mutant mice and propylthiouracil-treated rat pups (Uziel et al. 1981; O'Malley et al. 1995; Li et al. 1999), such abnormalities appear sporadically, and the variable nature of their expression suggests that the primary pathology may be unrelated to stereociliary bundles per se. This is particularly evident in the work of O'Malley and colleagues (O'Malley et al. 1995; Li et al. 1999), in which an apparent mismatch between the appearance of morphological defects and functional deficits is notable, i.e., morphological anomalies were variably scattered along the length of the cochlear spiral, while auditory brainstem response threshold-frequency curves revealed no evidence of discrete pathological foci. Furthermore, the finding that mechanoelectrical transduction currents in both inner and outer hair cells are normal in hearing-impaired TR $\beta$-deficient mice (Rusch et al. 1998) supports the view that stereocilia appear to function normally in animals with thyroid system abnormalities.

As for the tectorial membrane, although the structure clearly participates in transduction, recent studies suggest that its role may be less clearly understood than heretofore suspected (Legan et al. 2000; Russell et al. 2007). For example, in $\alpha$-tectorindeficient mice, although affected animals are insensitive to tonal stimulation, frequency tuning remains sharp even though the TM is detached from the spiral 


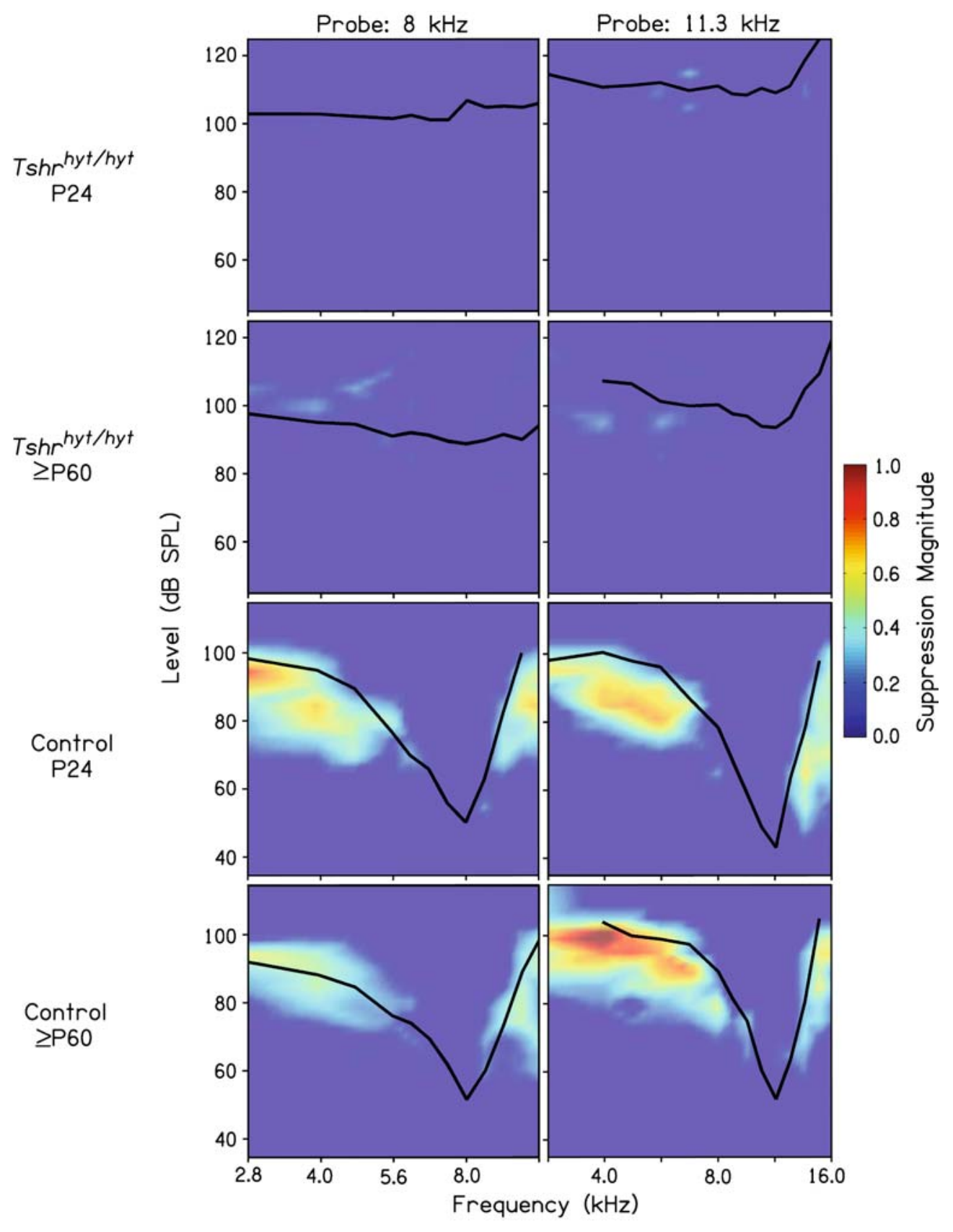

FIG. 5. Two-tone suppression contours (color-coded) are shown in relation to tuning curves (dark lines) for hypothyroid mice at P24 (top) and $\geq \mathrm{P} 60$ (middle) at $8 \mathrm{kHz}$ (left column) and $11.3 \mathrm{kHz}$ (right column). For comparison, the suppression contours and tuning curves for the $\geq \mathrm{P} 60$ agematched control groups are also shown (bottom). Suppression magnitude is plotted as the fraction of the probe-elicited response that was released from masking by the suppressor tone. The data shown are averages; the number of animals contributing to the averages was, from top to bottom, four, five, five, and four in cases shown in the left column and three, four, five, and five in cases shown in the right column.

limbus according to Legan et al. (2000). In the case of $\beta$-tectorin-deficient mice, although the second, lowfrequency resonance reported by Legan et al. (2000) was not observed, Russell et al. (2007) reported that high-frequency tuning sharpness is actually enhanced at $\mathrm{CF}$ relative to normal animals even though the TM is enlarged and exhibits the disrupted striated-sheet matrix described previously in hypothyroid rats that also exhibit reduced levels of $\beta$-tectorin (Knipper et al. 2001). While it is important to replicate these findings and to determine if they generalize to other models, these data generally suggest that the link between TM structure and composition and the sharpness of tuning may be more complex than previously thought. In addition, and perhaps most important, normal TM morphology has been observed in a variety of animal models in which auditory deficits have been associated with wide-ranging thy- 
roid system abnormalities that include frank congenital hypothyroidism and $\mathrm{TH}$ receptor mutations (Forrest et al. 1996; Li et al. 1999; Shibusawa et al. 2003), as well as in those cases in which functional deficits persist when TM defects have been rescued by thyroid hormone replacement (Deol 1973; Uziel et al. 1985; Ng et al. 2004). Consistent with these findings, preliminary data from our laboratory suggests that the TM's gross morphological features are normal in $T s h r^{h y t}$ mutant mice, except for the apical end of the cochlea of hypothyroid pups of hypothyroid dams, i.e., the TM appears normal even under conditions of extreme TH deficiency along most of its spiral length (Walsh and McGee 2001; Walsh et al. 2004, 2007). In this light, it is interesting that unlike the TM of propylthiouracil-treated rat pups that show relatively clear lectin abnormalities (Prieto et al. 1990; GilLoyzaga et al. 1990), lectin expression and distribution patterns in the TM of $T s h r^{h y t}$ mutant mice appear normal (Perales et al. 2002).

Finally, the arguments posed here that led us to discount the contribution of the middle ear as an etiological factor in hypothyroidism-induced otopathology apply equally in the case of other elements of transduction that affect passive, as well as active, aspects of transduction, for example the stria vascularis and by inference the endocochlear potential (EP). These elements can be largely dismissed as major factors contributing to the pathology observed in many forms of thyroid-related disease, because reductions in the EP necessarily diminish inner hair cell output and thereby affect passive aspects of transduction reflected in tuning curve tail thresholds, in addition to active elements (Sewell 1984). Although EPs are reduced in extreme cases of hypothyroidism or when $\alpha 1$ - and $\beta$-thyroid hormone receptors are absent (Rusch et al. 2001; Karolyi et al. 2007), normal EPs have been reported in TRß-deficient mice (Rusch et al. 1998), even though ABR thresholds are significantly elevated (Forrest et al. 1996).

The unanswered question lying at the center of this discussion then becomes, what is the underlying source of cochlear amplifier malfunction in Tshr mutant mice? On the surface, the parsimonious answer may be the OHC, a conclusion indirectly supported by the finding that tuning curve tails are normal and tuning curve tips are abnormal in hypothyroid animals and that distortion product otoacoustic emission thresholds are elevated in hypothyroid rodents ( $\mathrm{Li}$ et al. 1999; Knipper et al. 2000; Walsh and McGee 2001; Karolyi et al. 2007). Additional support can be found in the observation that two-tone suppression, a transduction nonlinearity clearly linked to normally functioning OHCs (Schmiedt et al. 1980; Dallos et al. 1980; Patuzzi et al. 1989; Geisler et al. 1990), was not observed in the responses of Tshr mutant mice. It nonetheless remains that the normal micromechanics of transduction depend on the orchestration of a complex chain of events requiring the precise interaction of multiple components, the development of which is under the control of a variety of trophic factors. Nevertheless, findings reported here suggest that it would be reasonable to begin the search for the source of hypothyroidism-induced otopathology in the domain of the OHC network.

\section{ACKNOWLEDGMENTS}

This research was supported by grants from the National Institute of Deafness and other Communication Disorders, DC00982, DC04566, DC04662.

\section{REFERENCES}

Anniko M, Rosenkvist U. Tectorial and basal membranes in experimental hypothyroidism. Arch. Otolaryngol. 108:218-220, 1982.

Brandt N, Kuhn S, Munkner S, Braig C, Winter H, Blin N, Vonthein R, Knipper M, Engel J. Thyroid hormone deficiency affects postnatal spiking activity and expression of $\mathrm{Ca} 2+$ and $\mathrm{K}+$ channels in rodent inner hair cells. J. Neurosci. 27:3174-3186, 2007.

Christ S, Biebel UW, Hoidis S, Friedrichsen S, Bauer K, Smolders JW. Hearing loss in athyroid Pax8 knockout mice and effects of thyroxine substitution. Audiol. Neurootol. 9:88-106, 2004.

Dallos P, Cheatham MA. Compound action potential (AP) tuning curves. J. Acoust. Soc. Am. 59:591-597, 1976.

Dallos P, Harris DM, Relkin E, Cheatham MA. Two-tone suppression and intermodulation distortion in the cochlea: effect of outer hair cell lesions. In: van den Brink G, Bilsen FA (eds) Psychophysical, Physiological and Behavioral Studies in Hearing. Delft, The Netherlands, Delft University Press, pp. 242-249, 1980.

Deol MS. An experimental approach to the understanding and treatment of hereditary syndromes with congenital deafness and hypothyroidism. J. Med. Genet. 10:235-242, 1973.

Deol MS. The role of thyroxine in the differentiation of the organ of Corti. Acta Otolaryngol. 81:429-435, 1976.

Fitzakerley JL, McGee J, Walsh EJ. Responses of peripheral auditory neurons to two-tone stimuli during development. I. Correlation with frequency selectivity. Hear. Res. 77:135-149, 1994a.

Fitzakerley JL, McGee J, Walsh EJ. Responses of peripheral auditory neurons to two-tone stimuli during development. II. Factors related to neural responsiveness. Hear. Res. 77:150161, $1994 \mathrm{~b}$.

Fitzakerley JL, McGee J, Walsh EJ. Responses of peripheral auditory neurons to two-tone stimuli during development. III. Rate facilitation. Hear. Res. 77:162-167, 1994c.

Forrest D, Erway LC, NG L, Altschuler R, Curran T. Thyroid hormone receptor beta is essential for development of auditory function. Nat. Genet. 13:354-357, 1996.

Gabrion J, Legrand C, Mercier B, Harricane MC, Uziel A. Microtubules in the cochlea of the hypothyroid developing rat. Hear. Res. 13:203-214, 1984.

Geisler CD, Yates GK, Patuzzi RB, Johnstone BM. Saturation of outer hair cell receptor currents causes two-tone suppression. Hear. Res. 44:241-256, 1990. 
Gil-Loyzaga P, Bueno AM, Broto JP, Perez AM. Effects of perinatal hypothyroidism in the carbohydrate composition of cochlear tectorial membrane. Hear. Res. 45:151-155, 1990.

Griffith AJ, Szymko YM, Kaneshige M, Quinonez RE, Kaneshige K, Heintz KA, Mastroianni MA, Kelley MW, Cheng SY. Knock-in mouse model for resistance to thyroid hormone (RTH): an RTH mutation in the thyroid hormone receptor beta gene disrupts cochlear morphogenesis. J. Assoc. Res. Otolaryngol. 3:279-288, 2002.

Gu WX, Du GG, Kopp P, Rentoumis A, Albanese C, Kohn LD, Madison LD, Jameson JL. The thyrotropin (TSH) receptor transmembrane domain mutation (Pro556-Leu) in the hypothyroid hyt/hyt mouse results in plasma membrane targeting but defective TSH binding. Endocrinology. 136:3146-3153, 1995.

HARRIS DM. Action potential suppression, tuning curves and thresholds: comparison with single fiber data. Hear. Res. 1:133-154, 1979.

Henley CM, Owings MH, Stagner BB, Martin GK, Lonsbury-Martin BL. Postnatal development of 2f1-f2 otoacoustic emissions in pigmented rat. Hear. Res. 43:141-148, 1990.

Johnson KR, Marden CC, Ward-Bailey P, Gagnon LH, Bronson T, Donahue LR. Congenital hypothyroidism, dwarfism, and hearing impairment caused by a missense mutation in the mouse dual oxidase 2 gene, Duox2. Mol. Endocrinol. 21:1593-1602, 2007.

Karoly IJ, Dootz GA, Halsey K, Beyer L, Probst FJ, Johnson KR, Parlow AF, Raphael Y, Dolan DF, Camper SA. Dietary thyroid hormone replacement ameliorates hearing deficits in hypothyroid mice. Mamm. Genome. 18:596-608, 2007.

Kiang NY, Liberman MC, Sewell WF, Guinan JJ. Single unit clues to cochlear mechanisms. Hear. Res. 22:171-182, 1986.

Knipper M, Zinn C, Maier H, Praetorius M, Rohbock K, Kopschall I, Zimmermann U. Thyroid hormone deficiency before the onset of hearing causes irreversible damage to peripheral and central auditory systems. J. Neurophysiol. 83:3101-3112, 2000.

Knipper M, Richardson G, Mack A, Muller M, Goodyear R, Limberger A, Rohbock K, Kopschall I, Zenner HP, Zimmermann U. Thyroid hormone-deficient period prior to the onset of hearing is associated with reduced levels of beta-tectorin protein in the tectorial membrane: implication for hearing loss. J. Biol. Chem. 276:39046-39052, 2001.

Legan PK, Lukashina VA, Goodyear RJ, Kossl M, Russell IJ, RICHARDSON GP. A targeted deletion in alpha-tectorin reveals that the tectorial membrane is required for the gain and timing of cochlear feedback. Neuron. 28:273-285, 2000.

Lenoir M, Puel JL. Development of 2f1-f2 otoacoustic emissions in the rat. Hear. Res. 29:265-271, 1987.

Li D, Henley CM, O’Malley BW, Jr. Distortion product otoacoustic emissions and outer hair cell defects in the hyt/hyt mutant mouse. Hear. Res. 138:65-72, 1999.

Liberman MC, Dodds LW. Single-neuron labeling and chronic cochlear pathology. III. Stereocilia damage and alterations of threshold tuning curves. Hear. Res. 16:55-74, 1984.

MeYerhoff WL. Hypothyroidism and the ear: electrophysiological, morphological, and chemical considerations. Laryngoscope 89:1-25, 1979.

Mills DM, Rubel EW. Development of the cochlear amplifier. J. Acoust. Soc. Am. 100:428-441, 1996.

Ng L, Goodyear RJ, Woods CA, Schneider MJ, Diamond E, Richardson GP, Kelley MW, Germain DL, Galton VA, Forrest D. Hearing loss and retarded cochlear development in mice lacking type 2 iodothyronine deiodinase. Proc. Natl. Acad. Sci. U. S. A. 101:3474-3479, 2004.

Norton SJ, Bargones JY, Rubel EW. Development of otoacoustic emissions in gerbil: evidence for micromechanical changes underlying development of the place code. Hear. Res. 51:7392, 1991.
O’Malley BW, Jr, Li D, Turner DS. Hearing loss and cochlear abnormalities in the congenital hypothyroid (hyt/hyt) mouse. Hear. Res. 88:181-189, 1995.

Patuzzi RB, Yates GK, Johnstone BM. Outer hair cell receptor current and sensorineural hearing loss. Hear. Res. 42:47-72, 1989.

Perales M, Segui D, Walsh EJ, Prieto JJ. Neurochemical abnormalities in the auditory pathway of the congenital hypothyroid ( hyt) hyt) mouse. Assoc. Res. Otolaryngol. Abstr. 25:167-168, 2002.

Prieto JJ, Rueda J, Sala ML, Merchan JA. Lectin staining of saccharides in the normal and hypothyroid developing organ of Corti. Dev. Brain. Res. 52:141-149, 1990.

Remezal M, Gil-Loyzaga P. Incorporation of D3H glucosamine to the adult and developing cochlear tectorial membrane of normal and hypothyroid rats. Hear. Res. 66:23-30, 1993.

Rubsamen R, Lippe WR. The development of cochlear function. In: Rubel EW, Popper AN, Fay RR (eds) Development of the Auditory System. New York, Springer, pp. 193-270, 1998.

Rusch A, Erway LC, Oliver D, Vennstrom B, Forrest D. Thyroid hormone receptor beta-dependent expression of a potassium conductance in inner hair cells at the onset of hearing. Proc. Natl. Acad. Sci. U. S. A. 95:15758-15762, 1998.

Rusch A, Ng L, Goodyear R, Oliver D, Lisoukov I, Vennstrom B, Richardson G, Kelley MW, Forrest D. Retardation of cochlear maturation and impaired hair cell function caused by deletion of all known thyroid hormone receptors. J. Neurosci. 21:9792$9800,2001$.

Russell IJ, Legan PK, Lukashkina VA, Lukashkin AN, Goodyear RJ, RichARDSON GP. Sharpened cochlear tuning in a mouse with a genetically modified tectorial membrane. Nat. Neurosci. 10:215223, 2007.

Schmiedt RA, Zwislocki JJ, Hamernik RP. Effects of hair cell lesions on responses of cochlear nerve fibers. I. Lesions, tuning curves, two-tone inhibition, and responses to trapezoidal-wave patterns. J. Neurophysiol. 43:1367-1389, 1980.

SEWELL WF. The effects of furosemide on the endocochlear potential and auditory-nerve fiber tuning curves in cats. Hear. Res. 14:305-314, 1984.

Shibusawa N, Hashimoto K, Nikrodhanond AA, Liberman MC, Applebury ML, Liao XH, Robbins JT, Refetoff S, Cohen RN, WONDISFORD FE. Thyroid hormone action in the absence of thyroid hormone receptor DNA-binding in vivo. J. Clin. Invest. 112:588-597, 2003.

Song L, McGeE J, WALsh EJ. Consequences of combined maternal, fetal and persistent postnatal hypothyroidism on the development of auditory function in $T s h r^{h y t}$ mutant mice. Brain Res. 1101:59-72, 2006a.

Song L, McGee J, Walsh EJ. Frequency- and level-dependent changes in auditory brainstem responses (ABRs) in developing mice. J. Acoust. Soc. Am. 119:2242-2257, 2006b.

Song L, McGeE J, WAlsh EJ. Development of cochlear amplification, frequency tuning and two-tone suppression in the mouse. J. Neurophysiol. 99(1):344-355, 2008.

Sprenkle PM, McGee J, Bertoni JM, Walsh EJ. Consequences of hypothyroidism on auditory system function in Tshr mutant (hyt) mice. J. Assoc. Res. Otolaryngol. 2:312-329, 2001 a.

Sprenkle PM, McGee J, Bertoni JM, Walsh EJ. Development of auditory brainstem responses (ABRs) in Tshr mutant mice derived from euthyroid and hypothyroid dams. J. Assoc. Res. Otolaryngol. 2:330-347, 2001b.

Sprenkle PM, McGee J, Bertoni JM, Walsh EJ. Prevention of auditory dysfunction in hypothyroid $T s h r$ mutant mice by thyroxin treatment during development. J. Assoc. Res. Otolaryngol. 2: 348-361, 2001c.

Stein SA, Oates EL, Hall CR, Grumbles RM, Fernandez LM, Taylor NA, Puett D, Jin S. Identification of a point mutation in the 
thyrotropin receptor of the hyt/hyt hypothyroid mouse. Mol. Endocrinol. 8:129-138, 1994.

TABERnER AM, Liberman MC. Response properties of single auditory nerve fibers in the mouse. J. Neurophysiol. 93:557-569, 2005.

Tubach M, McGee J, Walsh EJ. Distortion generated by the ear: its emergence and evolution during development. Laryngoscope 106:822-830, 1996.

Uziel A, Gabrion J, Ohresser M, Legrand C. Effects of hypothyroidism on the structural development of the organ of Corti in the rat. Acta Otolaryngol. 92:469-480, 1981.

Uziel A, Legrand C, Ohresser M, Marot M. Maturational and degenerative processes in the organ of Corti after neonatal hypothyroidism. Hear. Res. 11:203-218, 1983a.

Uziel A, Pujol R, Legrand C, Legrand J. Cochlear synaptogenesis in the hypothyroid rat. Brain Res. 283:295-301, 1983b.

Uziel A, Legrand C, Rabie A. Corrective effects of thyroxine on cochlear abnormalities induced by congenital hypothyroidism in the rat. I. Morphological study. Brain Res. 351:111-122, 1985.

Walsh EJ, McGee J. Hypothyroidism in the Tshr mutant mouse. In: Willott JF (ed) Handbook of Mouse Auditory Research: From Behavior to Molecular Biology. Boca Raton, CRC, pp. 537-555, 2001.

WaLsh EJ, Romand R. Functional development of the cochlea and the cochlear nerve. In: Romand R (ed) Development of Auditory and Vestibular Systems 2. Amsterdam, Elsevier, pp. 161-219, 1992.
Walsh EJ, McGee J, Javel E. Development of auditory-evoked potentials in the cat. III. Wave amplitudes. J. Acoust. Soc. Am. 79:745-754, 1986.

Walsh EJ, McGee J, Dallos P, He DZZ, Knipper M, Jia S, Zheng J. Prestin expression and electromotility in genetically-induced hypothyroidism. Assoc. Res. Otolaryngol. Abstr. 26:126, 2003.

Walsh EJ, Warr WB, Boche JE, McGee J. The organ of Corti and efferent olivocochlear innervation in congenitally hypothyroid (Tsh $r^{h y t}$ mutant) mice. Assoc. Res. Otolaryngol. Abstr. 27:1468, 2004.

Walsh EJ, Warr WB, Boche JE, McGee J. Cochlear morphology is delayed in Tshr (hyt) mutant hypothyroid mice. Assoc. Res. Otolaryngol. Abstr. 30:575, 2007.

Weber T, Zimmermann U, Winter H, Mack A, Kopschall I, Rohbock K, ZENNER HP, KNIPPER M. Thyroid hormone is a critical determinant for the regulation of the cochlear motor protein prestin. Proc. Natl. Acad. Sci. U. S. A. 99:2901-2906, 2002.

Winter H, Braig C, Zimmermann U, Geisler hS, Franzer JT, Weber T, Ley M, Engel J, Knirsch M, Bauer K, Christ S, Walsh EJ, McGee J Kopschall I, Rohbock K, KNIPPER M. Thyroid hormone receptors TRalpha1 and TRbeta differentially regulate gene expression of Kcnq 4 and prestin during final differentiation of outer hair cells. J. Cell. Sci. 119:2975-2984, 2006.

Winter H, Braig C, Zimmermann U, Engel J, Rohbock K, Knipper M. Thyroid hormone receptor alphal is a critical regulator for the expression of ion channels during final differentiation of outer hair cells. Histochem. Cell. Biol. 128:65-75, 2007. 